\title{
Surgical treatment of congenital left ventricular diverticulum
}

\author{
Minghui Yao, Rong Wang, Weihua Ye, Chonglei Ren \\ Department of Cardiovascular Surgery, the First Medical Center of PLA General Hospital, Beijing, China \\ Contributions: (I) Conception and design: M Yao; (II) Administrative support: C Ren; (III) Provision of study materials or patients: R Wang; (IV) \\ Collection and assembly of data: W Ye; (V) Data analysis and interpretation: M Yao; (VI) Manuscript writing: All authors; (VII) Final approval of \\ manuscript: All authors. \\ Correspondence to: Chonglei Ren. Department of Cardiovascular Surgery, the First Medical Center of PLA General Hospital, Fuxing Street 28, \\ Beijing 100853, China. Email: renchonglei301@163.com.
}

Background: Congenital left ventricular diverticulum (LVD) is a rare cardiac malformation. Its prevalence rate is less than $0.1 \%$ of the congenital heart diseases requiring surgery. Some scholars suggest that all LVD should be actively removed to prevent possible risks, including diverticulum rupture, arterial embolism, and malignant arrhythmia. However, others believe that asymptomatic LVD can be followed up without immediate surgery. We reviewed and reported the diagnosis, clinical features, and surgical treatment of four cases of congenital LVD to provide clinical experience and a reference for the treatment of such patients.

Methods: Four patients (aged 3-32 years old) were diagnosed with congenital LVD and received surgical treatment at the Department of Cardiovascular Surgery of PLA General Hospital, Beijing, China from September 2009 to July 2019. All four patients had complete long-term postoperative follow-up data, including echocardiogram, enhanced cardiac computed tomography (CT), and electrocardiogram to monitor changes in left ventricular structure, heart function, and heart rhythm.

Results: In the first case, the fibrodiverticulum under the aortic valve squeezed the right ventricular outflow tract and the right main coronary artery; the morphology of the right ventricle and coronary artery returned to normal after surgery. The second patient was complicated with a huge lipoma in the apex of the left ventricle and underwent lipoma resection during LVD resection surgery. The third and fourth cases had muscular diverticula in the left ventricular apexes and received LVD removal surgery. All four patients recovered well after surgery and their left ventricular morphology and cardiac function were normal without adverse complications, such as atrial fibrillation, ventricular arrhythmia, and cerebrovascular accident.

Conclusions: Although the morphology and character of congenital LVD were different in each case, the use of effective diagnostic and follow-up tools, including echocardiogram, enhanced CT, and magnetic resonance imaging (MRI), allowed for successful surgical treatment of the left ventricular diverticula and symptoms or other malformations. We propose that congenital LVD should be actively treated with surgery, especially considering effectiveness and low risk associated with this therapeutic option.

Keywords: Left ventricular diverticulum (LVD); lipoma; cardiac surgery

Submitted Nov 04, 2020. Accepted for publication Jan 12, 2021.

doi: $10.21037 /$ jtd-20-3510

View this article at: http://dx.doi.org/10.21037/jtd-20-3510

\section{Introduction}

Congenital left ventricular diverticulum (LVD) is a rare cardiac malformation that is caused by the reduction or deletion of local myocardial tissue or the replacement of normal myocardial tissue by fibrous tissue, resulting in abnormal bulging of myocardial tissues under high left ventricular pressure. The prevalence rate of congenital LVD is less than $0.1 \%$ of the congenital heart diseases requiring surgery (1). However, it has been reported that three cases of isolated LVD were found in a group of 750 patients after sudden cardiac death (0.4\%) (2). It is currently believed that it occurs as a result of impaired development 
of the endocardial tube during the 4th week of embryologic development. Some scholars suggest that all left ventricular diverticula should be actively removed to prevent possible risks, such as diverticulum rupture, arterial embolism, and malignant arrhythmia. However, other scholars believe that asymptomatic LVD can be followed-up without immediate surgery. Considering its low incidence rate, it is difficult to perform large-scale data analytics on cases of congenital LVD. In order to provide clinical experience and a reference for the treatment of such cases, the data of four congenital LVD patients found in our hospital from the past decade are described in the present study.

We present the following article in accordance with the AME Case Series reporting checklist (available at http:// dx.doi.org/10.21037/jtd-20-3510).

\section{Methods}

\section{Study subjects}

A retrospective study was conducted on a total of four patients who received LVD resection surgery (aged 3, 21, 22, and 32 years) in our hospital from September 2009 to July 2019. This study was conducted in accordance with the Declaration of Helsinki (as revised in 2013). The study was approved by the Institutional Ethics Committee of the First Medical Center of PLA General Hospital. Informed consent was obtained from all patients, and all personal data have been protected and secured according to current national and international laws.

\section{Inclusion criteria}

All patients were diagnosed as LVD by echocardiography, cardiac computed tomography (CT) enhanced scan, and magnetic resonance imaging (MRI) prior to surgery. The patients also had complete data of the left ventricular morphology, cardiac function, and heart rhythm examined by enhanced cardiac CT, echocardiogram, and electrocardiogram during the postoperative follow-up period.

\section{Statistical analysis}

Descriptive statistics was used to describe the cases.

\section{Results}

\section{Case 1}

A 3-year-old boy was admitted to our hospital with "repeated chest tightness and chest pain for half a year". The echocardiography and enhanced cardiac CT carried out in our hospital showed that there was a diverticulum with dimensions of approximately $3 \mathrm{~cm} \times 3 \mathrm{~cm}$ that was $1 \mathrm{~cm}$ below the right coronary sinus of the aorta in the left ventricle. Preoperative CT showed that the diverticulum protruded into the right pericardial cavity and squeezed the right ventricle, causing the right ventricle to shift to the left. The diverticulum was adjacent to the right coronary artery trunk and squeezed the right main coronary artery to the left (see Figure 1). Considering that the symptoms of repeated chest tightness and chest pain might be related to myocardial ischemia and right ventricular dysfunction caused by diverticulum compression on the coronary artery and the right ventricle, surgical treatment was decided.

Intraoperative exploration further confirmed the imaging diagnosis. The LVD was located under the right coronary sinus at the root of the aorta, close to the right coronary sinus. The diverticulum protruded to the right, squeezing the right atrium to the lower right and squeezing the right ventricle and the right coronary artery to the left, which resulted in changes in the shape and position of the right ventricle. After cardiopulmonary bypass was established, the aorta was opened transversely and the base of diverticulum was opened longitudinally. This revealed that the inner membrane of the diverticulum was a fiber-like structure, the size of the diverticulum was approximately $3 \mathrm{~cm} \times 3 \mathrm{~cm}$, and the outer layer was wrapped by muscular tissue. The neck of diverticulum located under the right coronary cups could be seen through aortic exploration, and the probe could pass through the aortic orifice to the incision at the base of the diverticulum. The neck of the diverticulum was closed with Decorn patch, double-ended needle, and spacer. Care was taken to prevent damage to right coronal cups and noncoronal cups. The base of the diverticulum that had been cut apart was repaired with two pieces of pericardium using the sandwich method to close the diverticulum cavity (see Figure 2). Postoperative cardiac CT showed that the LVD disappeared and the left and right ventricular morphology returned to normal (see Figure 3).

\section{Case 2}

A 21-year-old man was admitted to our hospital with "repeated palpitation for two months". The patient had palpitations after physical activities 2 months prior to admission, accompanied by chest tightness and shortness of breath, which was relieved after rest. The symptoms 



Figure 1 CT images of the LVD. (A) Coronal view; (B) sagittal view; (C) three-dimensional reconstruction showing that the LVD was under the aortic valve, adjacent to non-coronal cups. LA, left atrium; LV, left ventricle; RV, right ventricle; AO, ascending aorta; LVD, left ventricular diverticulum.
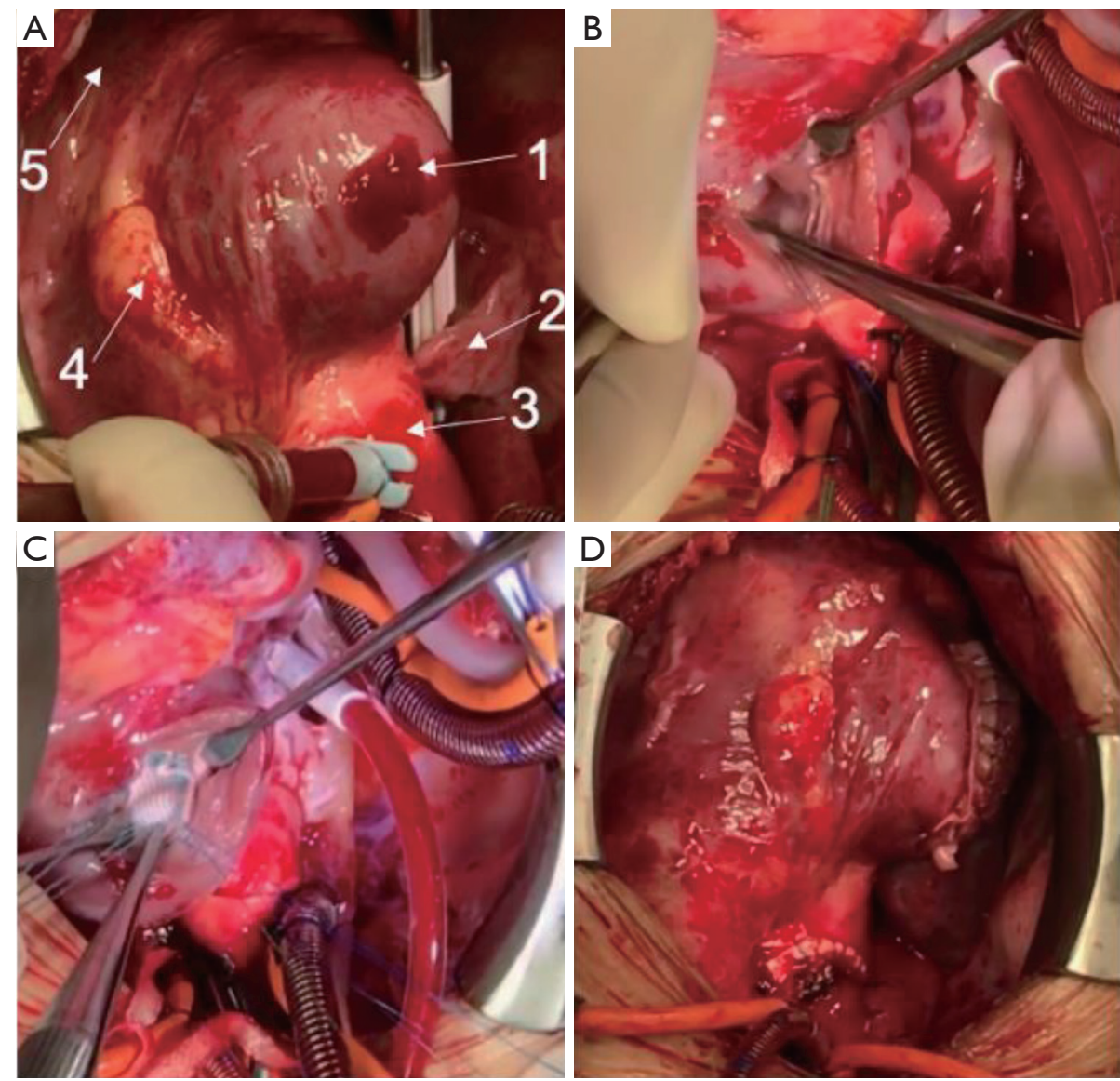

Figure 2 Surgical procedures of the LVD. (A) Exploration before cardiopulmonary bypass, [1] LVD, [2] right atrial appendage, [3] ascending aorta, [4] right coronary artery, [5] right ventricle; (B) incision and exposure of the diverticulum neck; (C) closure of the diverticulum neck with Decorn patch; (D) closure of the diverticulum cavity with two pieces of pericardium using the sandwich method. LVD, left ventricular diverticulum. 



Figure 3 Postoperative view after resection and closure of the LVD. (A) Coronal view of enhanced cardiac CT showing that the morphology and structure of all cardiac chambers were normal; (B) sagittal view of enhanced cardiac CT showing that the LVD disappeared and the left ventricular morphology returned to normal. LVD, left ventricular diverticulum; RA, right atrium; RV, right ventricle; LA, left atrium; LV, left ventricle; $\mathrm{AO}$, ascending aorta.

continued thereafter. One week before admission, the patient had palpitations in resting state. ECG after admission indicated sinus tachycardia, atrial arrhythmia, and premature ventricular contractions. Echocardiography showed that there was a $4 \mathrm{~cm} \times 3 \mathrm{~cm}$ limited bluge with the base $2 \mathrm{~cm}$ wide in the left ventricular apex, and an $8 \mathrm{~cm} \times 4 \mathrm{~cm}$ slightly hyperechoic solid mass in the pericardial cavity of the left ventricular apex. Diagnosis of the diverticulum of the left ventricular apex combined with lipoma of the left ventricular apex was made by further cardiac CT and MRI (see Figure 4). Since the patient repeatedly had palpitation symptoms after physical activities, ECG showed arrhythmia. LVD combined with a huge apical lipoma was diagnosed; the surgical indications were clear and surgical treatment was performed.

Intraoperative exploration showed that the diameter of the lipoma was about $8 \mathrm{~cm} \times 5 \mathrm{~cm}$, and the base of the lipoma was about $5 \mathrm{~cm} \times 3 \mathrm{~cm}$. Under cardiopulmonary bypass and cardiac arrest, the lipoma was free and completely resected along its base of the lipoma using an electrotome. At the same time, the diverticulum in the left ventricular apex was opened to expose the diverticulum cavity and the neck. It was found that the diverticulum was surrounded by muscle tissue. The papillary muscles and mitral valve leaflets in the anterior and posterior groups of the mitral valve could be detected through the neck of the diverticulum. The neck of diverticulum was closed with Decorn patch. The diverticulum cavity of the left ventricle was closed and repaired using the sandwich method. Since the base of the lipoma was wide, Decorn patches were used to cover the base of the lipoma and the diverticulum cavity to prevent tissue avulsion due to excessive suture tension, which would lead to bleeding. After the operation, the patient recovered satisfactorily. The echocardiography showed that the left ventricle was normal, the diverticulum disappeared, and no abnormal echocardiography morphology was found in the pericardium. ECG showed sinus rhythm and no adverse surgical complications. The patient recovered and was discharged (see Figure 5).

\section{Case 3}

A 22-year-old female patient was admitted to the hospital due to "enlargement of the heart for 5 years and chest tightness for 1 month". Echocardiography showed a solid mass in the pericardial cavity in front of the left ventricle. The mass cavity communicated with the left ventricular apex through a $3-\mathrm{cm}$ wide opening. Doppler flow imaging showed that the liquid in the mass was blood. The LVD was confirmed by CT angiography (CTA). The LVD was resected under cardiopulmonary bypass. Histopathological examination showed that the sample sent for examination was myocardial tissue and some myocytes were granular and exhibited vacuolar degeneration. After the operation, the echocardiographic structure and function of the heart were normal. The patient recovered and was discharged. 

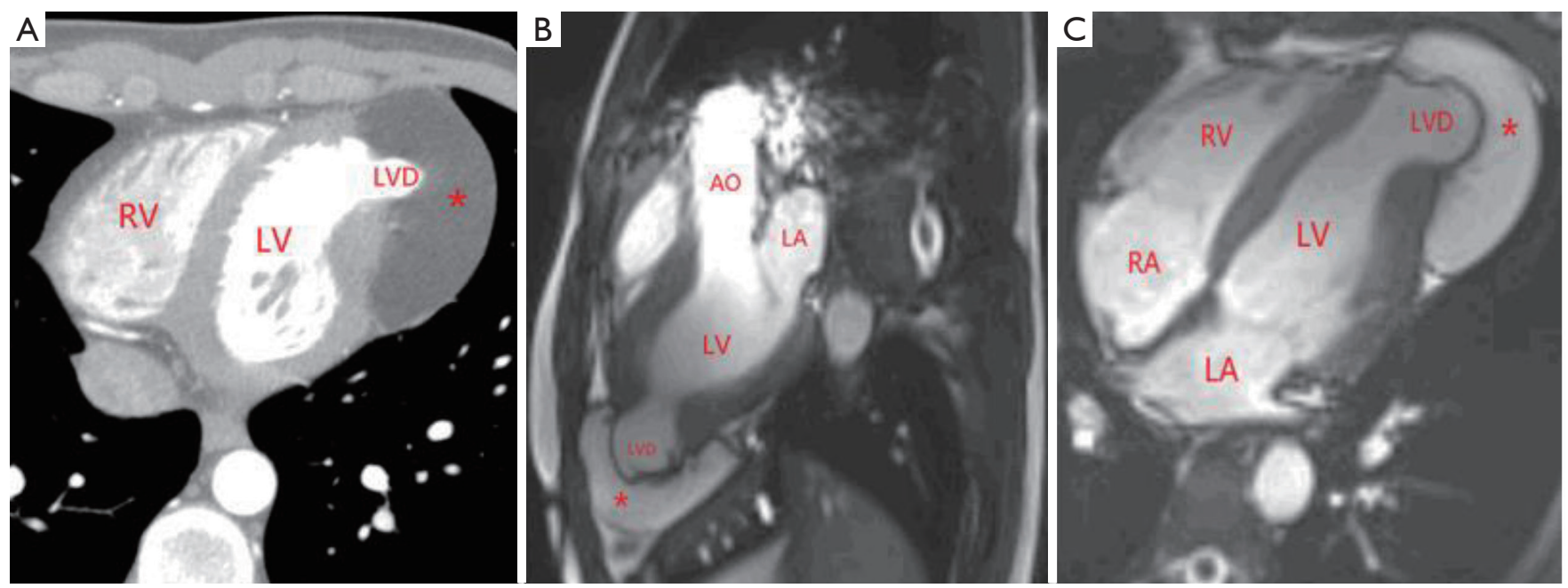

Figure 4 Images of the LVD with the left ventricular apical lipoma. (A) Coronal view of contrast-enhanced cardiac CT; (B) sagittal view of enhanced cardiac magnetic resonance; (C) coronal view of enhanced cardiac magnetic resonance. *, lipoma. RA, right atrium; RV, right ventricle; LA, left atrium; LV, left ventricle; AO, ascending aorta; LVD, left ventricular diverticulum.

\section{Case 4}

A 32-year-old female was hospitalized with "repeated palpitations for half a year". 24-hour Holter monitoring showed sinus rhythm with frequent ventricular premature (ventricular rhythm accounted for 7.2\%). Echocardiography showed that there was a $3 \mathrm{~cm} \times 3 \mathrm{~cm}$ limited bluge in the left ventricular apex. The wall of the bluge had synchronous motion with the left ventricular contraction. Enhanced cardiac CT conformed LVD and it was resected under cardiopulmonary bypass (refer to case 2 for surgical procedures). After the surgery, the echocardiographic structure and function were normal (Figure 6) and the ECG showed sinus rhythm without ventricular arrhythmia. The patient recovered and was discharged.

\section{Postoperative follow-up}

All four patients were reexamined at 3 months, 6 months, 1 year, and 2 years in postoperative follow-ups, respectively, and the follow-up rate was $100 \%$. The longest follow-up time was 11 years. During the follow-up period, there were no thromboembolic events. ECG showed no arrhythmia. Enhanced cardiac CT showed normal morphology and structure. And there were no hospital readmissions due to cardiac complications, and no other adverse events. During the follow-up, the ECG of Case 1 showed an incomplete right bundle branch block, however no diverticulum was found in echocardiography and enhanced cardiac CT, and the patient had normal left ventricle shape and function.

\section{Discussion}

Since O'Bryan (3) first described the LVD as a rare cardiac malformation in 1838 , there have been only few reports on LVD, and most of them are found in neonates and children. However, LVDs have also been found in different age groups (4). Since 2009, four cases of LVD (including one child and three adults) have been diagnosed and successfully treated in the Department of Cardiovascular Surgery of the PLA General Hospital. For this kind of rare disease, we completely recorded the image data of two patients with LVD before surgery, including left ventricular angiography, enhanced cardiac CT, and echocardiography. During the operation, we used a camera system to record the morphology of the LVD, as well as the process of resection and suture. The preserved images, including the enhanced cardiac CT and echocardiogram, are an important reference and theoretical basis, and have significance value for the clinical analysis of LVD.

The most common histological types of LVD are fibrous and muscular. Fibrous diverticulum is mostly located under aortic valve or mitral valve, the diverticulum wall was thin and without contractile function, and accompanied with little other cardiac or extracardiac malformations. In this case report, one case was a simple fibrous diverticulum located under the aortic valve, and was resected on due to 

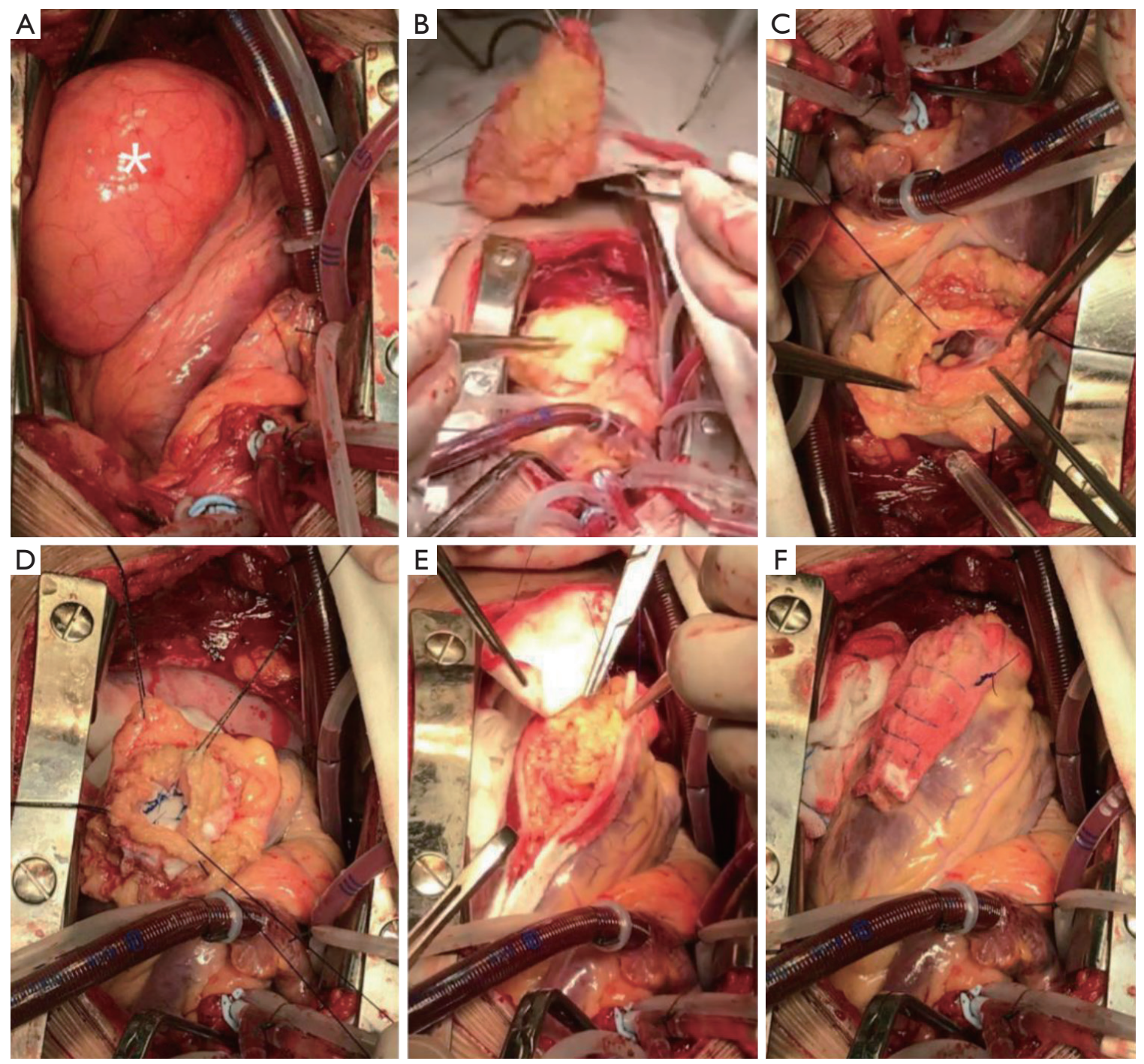

Figure 5 Surgical procedures for the LVD combined with the giant lipoma in the left ventricular apex. (A) Exposure and exploration of the apical lipoma after cardiac arrest; (B) incision along the median of the lipoma and excision of the lipoma layer by layer; (C) exposing and cutting the base of the diverticulum apart to expose the neck of the diverticulum; (D) repairing and closing the neck of the diverticulum with Decorn patch; (E) closure of the diverticulum cavity with felt and Decorn patch using the sandwich method; (F) image after closing the left ventricle. *, the morphology returns to normal. LVD, left ventricular diverticulum.

compression on the right ventricular outflow tract and the right coronary artery. Muscular LVD is often located in the apex of left ventricle, and contains three layers of myocardial structure, forming a real cavity and communicating with the left ventricular cavity through a short neck. The diverticulum wall can move synchronously with the normal ventricle wall. In the diastolic period, blood enters the diverticulum from the left ventricle, and the diverticulum becomes larger, while in systolic period, the blood flows from the diverticulum back to the left ventricle, and the diverticulum becomes smaller. This type of diverticulum is often associated with other cardiac malformations or thoracoabdominal malformations, such as ventricular septal defect, coronary artery malformation, or Cantrell syndrome (5). In this study, one case of left ventricular muscular diverticulum complicated with giant lipoma at apex of the heart, compressed the left ventricle, resulting in obvious palpitation and short of breath after activity. Preoperative ECG indicated ventricular arrhythmia, which was disappear postoperative. 

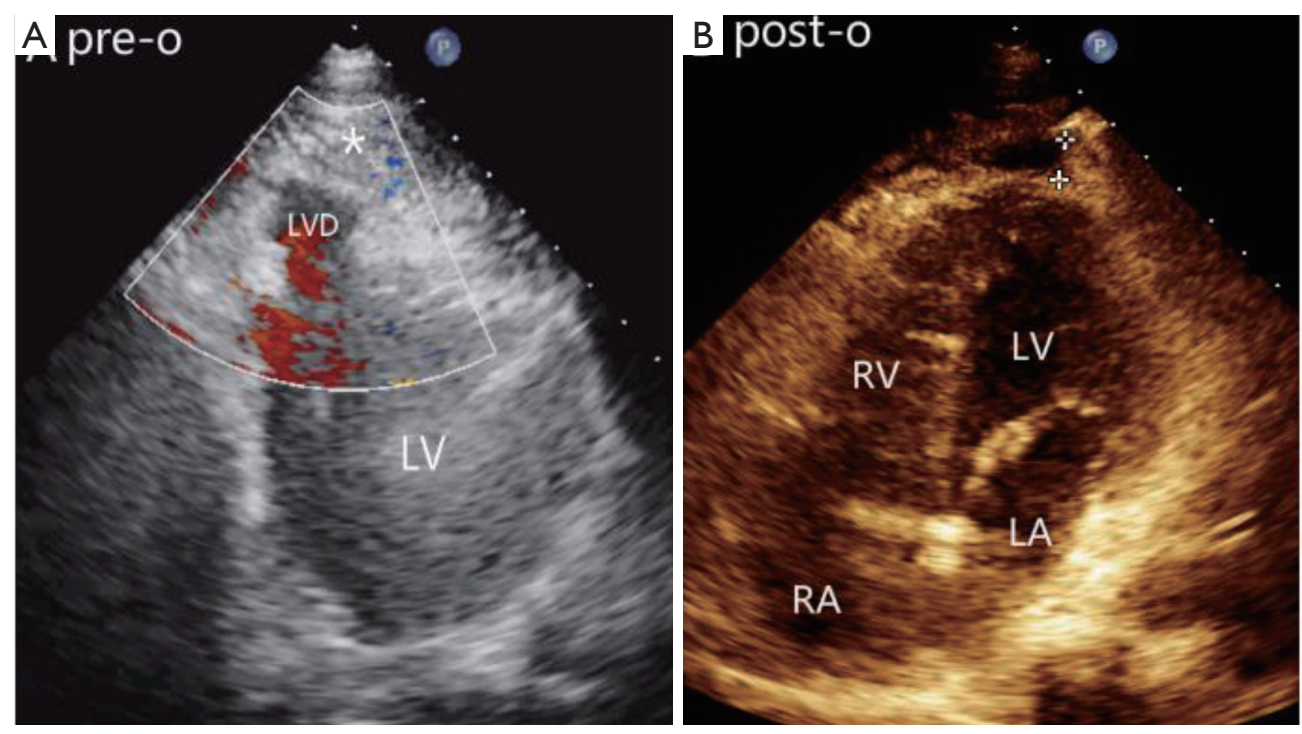

Figure 6 Preoperative and postoperative echocardiography of the LVD combined with the giant lipoma in the left ventricular apex. (A) Preoperative echocardiography; (B) no diverticulum or lipoma were found in postoperative ultrasonography. *, lipoma. LV, left ventricle; LVD, left ventricular diverticulum.

At present, malformation correction combined with LVD resection at the same time has been widely recognized for LVDs with symptoms or with cardiac malformations; however, there is still no consensus on whether or not to perform surgical treatment for asymptomatic isolated LVDs (6). Some scholars believe that all LVD patients require surgical treatment in order to reduce potential risks such as left ventricular thrombosis, malignant arrhythmia, ventricular wall rupture, and massive hemorrhage caused by LVD. However, others believe that radical surgical treatment of all LVD may cause damage to the left ventricular structure due to the resection of normal left ventricular wall muscles, which can result in heart failure and even lead to death. In this study, a case of simple left ventricular muscular diverticulum was included due to the enlargement of heart shadow during regular follow-up. Consideration that this patient was young, had a long life expectancy, and low surgical risk, surgical resection was performed to avoid the risk of left ventricular thrombosis, rupture, and arrhythmia caused by LVD. Our experience combined with previous literature reports makes us believe that LVD resection with cardiac malformation correction at the same time have low surgical risk and good effect for the LVD with other cardiac malformations, which is worthy of promotion. For simple muscular LVDs accompany with symptoms, such as compression of outflow tract, ventricular wall and coronary artery, or for gradually expanding LVD, surgical treatment should be considered. Surgical treatment should be also considered for the fibrodiverticulum because it is usually located under the aortic valve or mitral valve, which affects the structure of the valve leaflets, leading to aortic and mitral valve insufficiency. In addition, because the base of the fibrodiverticulum is usually wider than that of muscular diverticulum and lacks contractile function, the fibrodiverticulum is more prone to rupture. Our recommendations are consistent with the views reported in some studies (7).

Diseases related to congenital left ventricular expansion and outgrowth include congenital LVD and congenital left ventricular aneurysm (LVA). Both are rare congenital heart diseases. It is important to differentiate them in clinical practice as they differ in histologic type, clinical characteristics, natural process, and treatment scheme. Compared with congenital LVD, congenital LVA belongs to congenital heart diseases with endocardium or myocardial layer dysplasia, and its basement is a wide cystic structure. Although the aneurysm wall is continue with the normal ventricular wall, the aneurysm wall is thin, without movement or with contradictory movement. Additionally, congenital LVA is usually not companied with other cardiac malformations. Cardiography is the gold standard for differentiating congenital LVD and LVA. Fibrodiverticulum is not contractile and it is difficult to distinguish from congenital LVA or pseudoaneurysm. Delayed enhancement 
MRI can be used to distinguish congenital LVD and LVA by observing the myocardial perfusion. In histology, the ventricular wall of congenital LVA contains integral endocardium and epicardium, but the myocardium is thin or disappeared.

This study provides a good reference value for the study of such diseases, however the sample size of this single center retrospective study is relatively small. Thus, further studies need to be conducted to obtain more meaningful clinical results.

\section{Conclusions}

Congenital LVD is a rare cardiac malformation with different morphology and feature. Echocardiography, enhanced cardiac CT, and magnetic resonance imaging are effective tools for diagnosis and follow-up. For LVD with symptoms or other malformations and fibrous LVD, the risk of surgery is low and the therapeutic effect of surgery is good.

\section{Acknowledgments}

Funding: None.

\section{Footnote}

Reporting Checklist: The authors have completed the AME Case Series reporting checklist. Available at http://dx.doi. org/10.21037/jtd-20-3510

Data Sharing Statement: Available at http://dx.doi. org/10.21037/jtd-20-3510

Conflicts of Interest: All authors have completed the ICMJE uniform disclosure form (available at http://dx.doi. org/10.21037/jtd-20-3510). The authors have no conflicts of interest to declare.

Ethical Statement: The authors are accountable for all aspects of the work in ensuring that questions related to the accuracy or integrity of any part of the work are appropriately investigated and resolved. This study was conducted in accordance with the Declaration of Helsinki (as revised in 2013). The study was approved by the Institutional Ethics Committee of the First Medical Center of PLA General Hospital. Informed consent was obtained from all patients, and all personal data have been protected and secured according to current national and international laws.

Open Access Statement: This is an Open Access article distributed in accordance with the Creative Commons Attribution-NonCommercial-NoDerivs 4.0 International License (CC BY-NC-ND 4.0), which permits the noncommercial replication and distribution of the article with the strict proviso that no changes or edits are made and the original work is properly cited (including links to both the formal publication through the relevant DOI and the license). See: https://creativecommons.org/licenses/by-nc-nd/4.0/.

\section{References}

1. Mardini MK. Congenital diverticulum of the left ventricle. Report of two unusual cases. Br Heart J 1984;51:321-6.

2. Okereke OU, Cooley DA, Frazier OH. Congenital diverticulum of the ventricle. J Thorac Cardiovasc Surg 1986;91:208-14.

3. O'Bryan W. On Malformations of the Human Heart, 2nd edition. London: Churchill and Sons, 1838.

4. Ueda T, Mizushige K, Yukiiri K, et al. Contrast harmonic power Doppler imaging of congenital ventricular diverticulum--a case report. Angiology 2001;52:357-9.

5. Sakabe K, Fukuda N, Fukuda Y, et al. Isolated congenital left ventricular diverticulum in an elderly patient that was identified because of an incidental finding during a complete medical checkup. Int J Cardiol 2008;125:e30-3.

6. Marijon E, Ou P, Fermont L, et al. Diagnosis and outcome in congenital ventricular diverticulum and aneurysm. J Thorac Cardiovasc Surg 2006;131:433-7.

7. Jiang B, Yang YF, Wang X, et al. An Isolated Congenital Left Ventricular Diverticulum with Mitral Valve Endocarditis. Heart Lung Circ 2016;25:e53-5.

(English Language Editor: A. Kassem)
Cite this article as: Yao M, Wang R, Ye W, Ren C. Surgical treatment of congenital left ventricular diverticulum. J Thorac Dis 2021;13(1):291-298. doi: 10.21037/jtd-20-3510 